\title{
"EL QUINTO, CALLADO; CUANDO MÁS, SÍ Y NO". PROPUESTA DE UN MÉTODO ANALÍTICO DE FIGURAS NARRATIVAS EJEMPLIFICADA EN SEGUIR DE POBRES DE IGNACIO ALDECOA
}

\author{
Kurt Spang \\ Universidad de Navarra
}

Sobra una justificación del interés y la valía de Ignacio Aldecoa como narrador; ya se considera uno de los clásicos modernos, como lo demuestran J. L. Martín Nogales' y otros estudiosos. Tampoco es preciso subrayar el valor literario, y no solamente literario, de $\mathrm{Se}$ guir de pobres ${ }^{2}$, uno de los mejores cuentos de la primera época, publicado en el volumen de Espera de tercera clase de 1955.

La capacidad de crear y evocar figuras es particularmente llamativa en la obra narrativa de Aldecoa y quizá aún más en los cuentos donde la necesidad de síntesis obliga a unas técnicas refinadas. Seguir de pobres se presta particularmente a un análisis de las figuras porque es un cuento en el que desempeñan un papel más destacado y, además, su número es suficientemente reducido como para poder analizar la totalidad. En este cuento, los segadores, y sobre todo El Quinto, a pesar de su aparente marginación y marginalidad, o quizá precisamente por cllo, son tratados con especial maestría y particular cariño, ese cariño reivindicativo, tan característico del autor alavés.

Para el análisis de las figuras consideraré, en primer lugar, los emisores de la información caracterizadora, es decir, las intervenciones del narrador y las réplicas de las propias fi-

1.- J. L. Martín Nogales, Los cuentos de Ignacio Aldecoa, Madrid, Cátedra, 1984.

2.- Cf. J. M. Martínez Cachero, "Ignacio Aldecoa: Seguir de pobres", El comentario de textos 2, Madrid, Castalia, 1974. 


\section{KURT SPANG}

guras en cuanto agentes de caracterización ${ }^{3}$. Además intentaré una clasificación de las figuras según el grado de individualización, según su evolución en el cuento y el peso que desempeñan en el desarrollo de la historia. En todos los enfoques, procuraré destacar la función expresiva que caracteriza cada uno de los recursos en el texto, también en el ámbito de las configuraciones, es decir, de las agrupaciones momentáneas e inestables de las figuras en las diversas secuencias, $y$, finalmente, en el reparto como totalidad de las figuras que pueblan el cuento.

Tal vez deba recordar brevemente el sencillo argumento de Seguir de pobres para situar a las figuras: una cuadrilla de cuatro segadores míseros en busca de trabajo se cruza en su camino con un hombre recién salido de la cárcel, que se junta al grupo con el nombre de "El Quinto". F́ste cae enfermo en un pueblo donde han encontrado trabajo, no puede seguir segando y despierta inmediatamente la solidaridad de sus compañeros que, al finalizar el cmpleo, le acompañan hasta la salida del pueblo y hasta le dan una parte de sus escasos ahorros, revelándose así mucho más "ricos" que los adinerados agricultores para quienes trabajaban.

\section{La cuadrilla y EI Quinto}

A lo largo del cuento se insiste reiterativamente en el contraste rico-pobre sugiriendo constantemente que esta oposición está vinculada ineludiblemente con el contraste solidaridad-insolidaridad. Las figuras del cuento encarnan esta oposición y en sus interacciones se revelan las cualidades de unos y otros. El autor trata al grupo de los segadores con más detalle y simpatía, enfrentándolo con el de los agricultores ricos a los que critica duramente. Sin embargo, tampoco es una confrontación torpemente antitética; al autor, ante todo, Ic interesa evocar la situación como consuetudinaria, rutinaria, aceptada resignadamente por los segadores y considerada por los agricultores como el estado natural de las cosas. La crítica social más dura, hábilmente insinuada, se dirige contra el inmovilismo de la sociedad en todos sus estamentos.

El primer contacto que el lector establece con el grupo de los segadores se realiza a través de una intervención del narrador, quien lo describe en camino en búsqueda de un trabajo:

En la cuadrilla van hombres solos. Cinco hombres solos. Dos del noroeste, donde un celemín de trigo es un tesoro. Otros dos de la parte húmeda de las Castillas. El quinto, de donde los hombres se muerden los dedos, lloran y es inútil (26)4.

Evidentemente, esta escueta pincelada es más enigmática que aclaratoria; crea clima más que caracteriza. En primer lugar, porque el narrador ni siquiera indica el nombre de las figuras y se limita a insinuar su procedencia evocando un ambiente de pobreza y soledad, y en el caso del Quinto se añade una nota de desesperación, tristeza e impotencia. Esta sugerencia puede parecer vaporosa y superflua, $y$, sobre todo, demasiado desprendida de las figuras como para servir de orientación. Sin embargo, es un primer connotador que orienta la lectura y predispone al receptor creando una expectación determinada, puesto que significa que las figuras de las que se va a hablar son seres desgraciados, dignos de nuestra compasión, lealtad e incluso de nuestro compromiso. Un poco más abajo, el narrador vuelve a insistir - en parte recogiendo literalmente algunas formulaciones-en estos detalles:

\footnotetext{
3.- Para la terminología empleada y adaptada a las necesidades narratológicas, véase el capítulo 4, "Figura y reparto", de mi Teoría del drama, Pamplona, Eunsa, 1991, pp. 155-197.

4.- Las citas se señalan con el número de la página entre paréntesis y se refieren a la recopilación Ignacio A/decoa, "Cuentos completos", edición de A. Bleiberg, Madrid, Alianza Fiditorial, 1973.
} 


\section{"EL QUINTO, CALLADO; CUANDO MÁS, SÍ Y NO"}

Cinco hombres solos. Cinco que forman un puño de trabajo. Dos del noroeste: Zito Moraña y Amadeo, el buen Amadeo, al que le salen barbas en el dorso de las manos, que se afeita con una hoz. Dos de la Castilla verde: San Juan y Concjo. El quinto, sin pucblo, del estaribel de Murcia por algo de cuando la guerra. El quinto, callado; cuando más, sí y no. El quinto, al que Ilaman desde que se les unió, sencillamente, "El Quinto", por un buen sentido nominador (26).

Se amplía, por tanto, la información sobre las figuras añadiendo los nombres de los cuatro que forman el grupo inicial y el detalle, no gratuito, de la "barba" en la mano de Amadeo. En cuanto al Quinto, se acentúa aún más la indeterminación de su procedencia a través de la puntualización "sin pueblo", que intensifica los aspectos que acabo de destacar; lo nucvo es el matiz de que estuvo en la prisión por algún delito relacionado con la guerra, delitos, al parecer, más fácilmente perdonables o incluso justificados, máxime si eran "cosa de hombre". Utilizar la voz popular "estaribel" equivale a una implícita atribución de la figura a una clase social baja y, por tanto, una identificación del narrador con el mundo de los segadores, y más directamente con el Quinto, una identificación y simpatía que se mantienen a lo largo de todo el cuento. Significativamente, se conserva la misma indeterminación en relación con la causa de su encarcelamiento -obsérvese la expresiva elipsis- "por algo de cuando la guerra" (26), que además es una especic de atenuante que disminuye la posible culpabilidad. Lo mismo se observa también en la reacción de los demás segadores. Esta vaga alusión a la guerra, de paso, sirve también de indicio para la ubicación temporal de la historia.

En esta misma intervención aparece el primer rasgo de carácter del Quinto, sugerido en una magistral elipsis y ejemplar adecuación estética de la expresión a la idea: "El quinto, caIlado; cuando más, sí y no". Pocas veces la taciturnidad se expresó con menos elementos y con más acierto literario. Como se comprobará en los demás elementos caracterizadores, la poca locuacidad es sólo el aspecto más llamativo y más palpable de un ser retraído, tímido, solitario e ingenuo. El hecho mismo de que los de la cuadrilla le llamen El Quinto, "por un buen sentido nominador", puede parecer nimio a primera vista, pero es, en realidad, lograda expresión de su anonimato y de su desvinculación del grupo c, implícitamente, de la sociedad. El detalle cobra más relieve porque contrasta con el final del cuento, donde, al despedirse, los cuatro segadores le dirán "Adiós, Pablo, adiós" (32), y el narrrador añadirá: "Hacía quince días que habían aprendido el nombre del "Quinto"” (32), es decir, se había producido una radical transformación de la relación entre los ahora compañeros 5 .

En la primera ocasión en la que El Quinto se dirige a sus futuros compañeros se produce una de las frecuentes retrospecciones de este cuento: “"El Quinto' les dijo en la cantina de la estación donde se lo tropezaron: '-Si van para el campo y no molesto voy con ustedes'” (26). Es una autocaracterización que reitera y subraya los rasgos que ya conocemos: la poca locuacidad, El Quinto va al grano y habla sin rodeos; pero además, asoma aquí un rasgo nuevo, su decisión, su personalidad a pesar de la timidez que acabamos de observar; no dice a los de la cuadrilla "Permitan que vaya con ustedes", sino "si (...) no molesto voy con ustedes". Es más, en la siguiente intervención del narrador se pormenoriza este rasgo -obsérvese la técnica cinematográfica del "travelling" con la que se realiza: el narrador siguiendo la mirada del Quinto como una cámara-, parece que quiere sugerir que, antes de

5.- No puedo reparar aquí en el tratamiento específico del tiempo que aplica Aldecoa en este cuento; habría que analizar las manipulaciones en la parte introductoria (pp. 25-27) con continuas retrospecciones, señaladas por los tiempos verbales y los saltos en el tiempo marcados por las líneas de puntos que separan las secuencias. 


\section{KURT SPANG}

juntarse a la cuadrilla, EI Quinto pretende asegurarse de que cada uno de los componentes, por separado, esté de acuerdo con su incorporación:

"El Quinto" movió la cabeza, clavó los ojos en Moraña, pasó la vista sobre Amadeo, que se rascaba las manos; consultó con la mirada a San Juan, que liaba un cigarillo parsimonioso sin que se le cayera una brizna de tabaco, y por fin miró a Conejo, que algo se buscaba en los bolsillos.

-Acabo de salir de la cárcel. ¿Qué dicen?

$-i Y$ usted? -respondió Zito.

-La guerra, y luego, mala conducta.

$-i$ Mala?

-De hombre, digo yo.

-Pues está dicho.

"El Quinto" pidió un cuartillo de vino tinto. La cita fue para las cinco y media de la mañana en el depuertas de la carretera. Se separaron.

Ahora los cinco van agrupados por el camino largo de los segadores. Zito conoce el terreno. Todos los años deja su tierra para segar a jornal.

-Amadeo, de la revuelta ésa nos salió el pasado una liebre como un burro.

-Sí, hombre; pero no el pasado, sino otro año atrás.

-Fue lástima...

Zito y Amadeo hablan del antaño perdiéndose en detalles, mientras San Juan se suena una y otra ve z la nariz distraídamente, mientras Conejo se queja de un murmullo de su alpargata rota, mientras "EI Quinto" va mirando los bordes del camino buscando no sabe qué (26).

Repárese cómo a través de la descripción de la forma de liar el cigarillo o de quejarse de la alpargata rota se logra mucho más que la mera descripción de la actuación de las figuras; es toda una historia de privaciones y renuncias que se esconde detrás de estas aptitudes y actitudes. Este tipo de breve pincelada que siempre se limita a la evocación de un rasgo externo a las figuras se disemina a lo largo de todo el cuento, sobre todo en la secuencia inicial que hace de exposición. El mismo hecho de no querer indagar en lo que cra aquel comportamiento "de hombre" con el que El Quinto justifica su "mala conducta", es de una fuerza caracterizadora extraordinaria. Connota subliminalmente una generosidad y capacidad de comprensión insólitas en la cuadrilla.

Sería interesante analizar más detenidamente la sintaxis del párrafo introductorio del fragmento citado; la unión de las oraciones refleja tipográficamente la unión de los cuatro que, por ahora, se enfrentan al quinto; hecho que ya se refleja en el uso del tratamiento de usted. En cambio, el laconismo del Quinto confirma lo que vimos ya en repetidas ocasiones, y se observa igualmente en los miembros de la cuadrilla en su relación con "el nuevo". Ahora abundan las oraciones lacónicas y la elipsis en las réplicas:

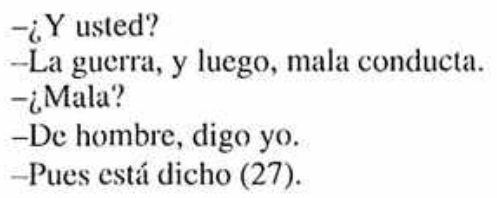

El rasgo externo del comportamiento del Quinto -“'El Quinto' va mirando los bordes del camino buscando no sabe qué" (27)- intensifica la impresión que ya sacamos de observaciones anteriores. Aumenta la impresión de marginación y aislamiento de la figura; su di- 


\section{"EL QUINTO, CALLADO; CUANDO MÁS, SÍ Y NO"}

ficultad de comunicarse y de establecer contacto se refleja hasta en su forma de andar. Y no es casual que nos lo comunique el narrador asumiendo el papel de observador, que contempla desde la distancia. Astutamente, Aldecoa no aprovecha en este cuento la posibilidad de convertirse en narrador omnisciente, sólo revela lo que puede registrar desde fuera, no se adentra en los pensamientos y sentimientos de la figura, respetando así su automarginación y destacando a la vez su aislamiento; lo juzga desde fuera y con la perspectiva de un narrador distanciado con un saber limitado. Zito Moraña, presentado en el cuento como una especie de cabeza y portavoz del grupo, "ve" la situación también así. El narrador, con simpatía, lo cvoca describiendo su capacidad de comprensión y compasión:

Aquí está Zito Moraña para preguntar, porque a un compañero hay que darle ocasión, sin molestarle, de un suspiro, de una lágrima, de una risa. Un compañero puede estar necesitado de descanso y es necesario saber, cuando cuente, el momento en que hay que balancear la cabeza o agacharla hacia el suelo o levantarla hacia el sol (28).

En el fondo, esta intervención presupone una introspección omnisciente, dado que se adentra en el pensamiento y carácter de Zito. Sin embargo, Aldecoa consigue formularla de tal modo que suena a observación general; hábilmente se limita en esta evocación a rasgos externos (suspiro, lágrima, risa, balancear la cabeza...) para sugerir sensaciones y estados anímicos. Sigue la misma técnica al reproducir el diálogo entre Zito y el Quinto:

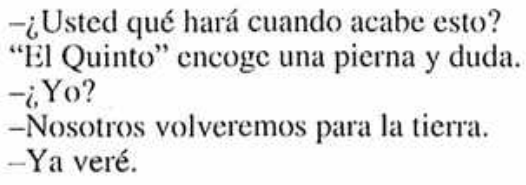

Y entre cllos, entre los cuatro y "El Quinto", el corazón de la comunidad naufraga $(28)^{6}$.

Nuevamente es de notar la manipulación de los pronombres personales: Zito trata de usted al Quinto y habla a continuación de "nosotros"; y el narrador remata la separación evocando la distancia "entre los cuatro y 'El Quinto', el corazón de la comunidad naufraga", siendo la "y" señal de disyunción más que de conjunción.

\section{Los labradores ricos}

Aldecoa aprovecha el final de la primera y larga secuencia para introducir un nuevo grupo de figuras, esta vez. en fuerte contraste con el ya conocido; es el de los agricultores ricos, que se opone de manera tajante al de los segadores. Para la evocación del conflicto y de la problemática del cuento, es preciso que haya contacto e interacción entre los dos grupos. Por ello, Aldecoa nos los presenta, desde ahora, en su precaria y distanciada convivencia efímera. Le interesa crear la sensación de que para los habitantes del pueblo los segadores son unos intrusos indeseables pero necesarios, y, por consiguiente, el trato con ellos es condescendiente y humillante. Veamos primero los rasgos que destaca $\mathrm{el}$ autor para presentar el grupo de los agricultores.

Tampoco en esta ocasión el autor se deja llevar por una facilona pintura en blanco y negro, por la distinción simplista de buenos y malos. Introduce matices que subrayan la impresión que mencioné ya al principio: su intención es criticar la naturalidad con la que ambos estamentos de la sociedad aceptan y con cllo confirman la situación como inamovible. Sin embargo, cabe preguntarse - como observación general-si Aldecoa no ha podido contemplar

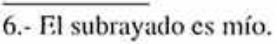




\section{KURT SPANG}

y juzgar con demasiado idealismo a los segadores como grupo social. Parece por lo menos dudoso que la virtuosidad que atribuye a los segadores del cuento tenga validez universal.

La situación se revela desde el primer momento. En la plaza del pueblo los segadores ya están tratados con cautela por los mismos niños, como si fuesen peligrosos y casi criminales. Evidentemente, esta actitud no corresponde a la reacción espontánea de los niños sino a lo que sus padres les inculcaron respecto de los segadores:

En la mitad [de la plaza] está el pilón; en él juegan los niños. Al verlos a los cinco parados y ensimismados, los niños se les acercan a una distancia de respeto y prudencia. Los segadores, como los gitanos, pueden robar criaturitas para venderlas en otros pucblos (28-29).

Ya en casa de Martín, el labrador que los contrató y les adjudicó un pajar para dormir, los segadores bajan para "ver si nos dan algo en la cocina" (29) y "les dan un trozo de tocino a cada uno, pan y vino" (29). Después, la mujer de Martín exige que Zito cante algo "de por tu tierra"(29); no le agrada la idea a Zito porque -hermosa perífrasis-tiene "la garganta con nudos"(29). Finalmente canta una copla que, además de dar título al cuento, resume concisamente la problemática central. Forma, por así decir, el núcleo del cuento. Canta así:

$$
\begin{aligned}
& \text { Al marchar a la siega } \\
& \text { entran rencores } \\
& \text { trabajar para ricos } \\
& \text { seguir de pobres. }
\end{aligned}
$$

Es la única ocasión en la que asoma por boca de los segadores un tono de crítica abierta; y no es una casualidad que esta crítica se atribuya a una voz ajena y anómina; Zito no es el autor de la copla, sólo es el intérprete y transmisor. Es más, para resaltar la ingenuidad de los segadores el narrador nos revela que, vueltos al pajar, su única preocupación es saber el importe de su escuetos jornales durante la estancia: "-Oye, San Juan, son unos viente días aquí. A doce pesetas, ¿cuánto viene a ser?" (30). Como en otras ocasiones, el autor aprovecha un detalle para añadir un toque caracterizador a sus figuras: el anómino preguntador -se nos revela subrepticiamente- es analfabeto y no domina las cuatro reglas.

Por si quedaban dudas acerca de la actitud de los agricultores frente a los recién venidos, el autor añade en esta misma secuencia una conversación entre Martín y un amigo en la que aquél, inconscientemente, se autocaracteriza hablando en términos inequívocos:

-Me han ofrecido material humano a siete pesetas para hacer toda la campaña, pero son andaluces...

-Gente floja.

-Floja.

Martín hace con los labios un gesto de menosprecio (30).

\section{Los segadores y los labradores ricos: una difícil convivencia}

A partir de este momento, el autor nos presenta a los dos grupos, segadores y agricultores, en distanciada coexistencia pasajera. Además se forman dos grupos de segadores; sin embargo, en comparación con el abismo que media entre segadores y agricultores, la distancia entre los que forman el quinteto ahora es externa y superficial, no tardará en empezar a nacer y consolidarse entre ellos "el corazón de la comunidad".

Trabajaban San Juan y Conejo con Martín. Zito Moraña, Amadeo y "El Quinto", con otros segadores que llegaron un día después, segaban en las fincas del alcalde. No se veían los dos grupos más que cuando marchaban al trabajo o volvían de él por los caminos. Zito, Amadeo y "El Quinto" dormían en el pajar del alcalde, 
sobre paja medio pulverizada. Se pasaban el día en el campo. (...) El viento pardo vino por el camino levantando una polvareda. Su primer golpe fue tremendo. Todos lo recibieron de perfil para que no les dañase, excepto "El Quinto", que lo soportó de espaldas, lejano en la finca, con la camisa empapada en sudor, segando. Le gritaron y fue inútil. No se apercibió. Cuando levantó la cabeza era ya tarde.

"El Quinto" llegó al pajar tiritando. Y no quiso cenar. Le dieron miel en las espaldas. El alcalde llamó al médico. El médico lo mandó lavar porque opinó que aquello eran tonterías. Y dictaminó.

-No es nada. Tal vez haya bebido agua demasiado fría.

Zito le explicó:

-Mire, doctor, fue el viento pardo...

El médico se cnfadó.

Cuanto más ignorantes, más queréis saber. ¿Qué me vas a decir tú?

-Mire doctor, fue el viento que mata el cereal y quema la yerba. Hay que darle de miel. Las mantecas de los riñones las tiene blandas.

- Bah, bah, el viento pardo... - coment6.

Los compañeros volvieron a darle miel en las espaldas en cuanto se marchó el médico, y Zito le echó su manta.

¿Y tú, Zito? -dijo "II Quinto".

Yo, a medias con Amadeo.

"El Quinto" temblaba; le castañeteaban los dientes. El viento pardo en el saucal hacía un murmullo de risas (30)-31).

Es ahora cuando crece - tal vez para acentuar aún más el contraste entre los dos grupos opuestos- la solidaridad entre los cinco segadores; no solamente fomentada por el trabajo, sino también por la enfermedad del Quinto causada - según los segadores- por el "viento pardo". En una cntrañable evocación, Aldecoa pinta la superstición de los segadores convencidos de que el maléfico "viento pardo" es el que ataca maliciosamente a la gente del campo y les produce fiebre y malestar. Los "entendidos", entre ellos, saben que el viento pardo se debe recibir de perfil y no de frente o de espaldas, como le ocurrió a El Quinto. No deja de ser un rasgo caracterizador que los segadores conozcan los remedios cascros para los que caen víctimas del viento pardo y que se fíen más de ellos que de la ciencia del médico.

Sin embargo, se debe contabilizar a favor de los agricultores ricos -y también a favor de Aldecoa- que se introduzca el detalle del aviso al médico al ponerse enfermo el Quinto. Una presentación simplista hubiera omitido el matiz aumentando así, de forma melodramática, la "maldad de los opresores". Resulta, sin embargo, revelador que Aldecoa mantenga una postura parcial ante la evidente superstición de los segadores. Tal es su simpatía que más le importa evocar su entrañable ingenuidad que juzgar el primitivismo o incluso la inutilidad de sus procedimientos. Es más, casi les da la razón a los segadores describiendo con seriedad sus actitudes y al hablar, concluyendo el episodio, del "murmullo de risas" del viento pardo.

La breve descripción de la pelea de la araña con una avispa, al principio de la siguiente secuencia, no solamente funciona como evocación de la ingenuidad del Quinto, también sirve de telón de fondo que recuerda la relación entre ricos y pobres. Fis precisamente ahora cuando renace "el corazón de la comunidad" que había "naufragado" caminando cl grupo hacia el pueblo. La comunidad y la solidaridad se instauran, primero, a través del ancestral tratamiento "médico", y luego, por el hecho de que Zito cede su manta a El Quinto, compartiendo a medias con Amadeo, y en la preocupación por el futuro del enfermo:

Allí estaba "El Quinto", entretenido con las arañas. Las iba conociendo. Contó a Zito y a $\Lambda$ madeo cómo había visto pelear a una de ellas, la de la gran tela, de la viga del rincón, con una avispa que atrapó. L co contaba infantilmente. Zito callaba. De vez en vez le interrumpía doblándole la manta. 


\section{KURT SPANG}

- ¿Qué tal ahora?

-Bien, no te preocupes.

- ¿No me he de preocupar? Has venido con nosotros y no te vas a poder marchar. Nosotros dentro de cuatro días tiramos para el norte. Esto está ya dando las boqueadas.

-Bueno, qué más da. No me echarán a la calle de repente...

-No, no, desde luego... - dudaba Zito.

-Y si me echan, pues me voy.

$-i$ Y adónde?

-Para la ciudad, al hospital, hasta que sane.

- Hum... (31-32).

No cabe duda de que El Quinto ya forma parte integrante del grupo y el Zito se siente tan responsable de él como de los demás. Debería comentarse igualmente la crítica social que implica tanto este diálogo como otras muchas partes del cuento7.

De nuevo se destaca el enfrentamiento tácito entre los dos grupos cuando el agricultor - a través de Zito y no personalmente ("Pues díselo y también que levante con vosotros")desaloja al enfermo de su casa. De modo que no cra infundada la duda de que los dueños fuesen a echar al enfermo del pajar antes de que sanara; se desprende claramente del diálogo entre Zito y el hipócrita terrateniente, que, para colmo, se considera muy generoso:

-Y dile al "Quinto" que para él, aunque no ha trabajado más que tres días y le he estado dando de comer todo este tiempo, hay diez duros. No se quejará.

-No, claro.

-Pues díselo y también que levante con vosotros.

-Pero si es imposible, si está tronzado.

-Y yo qué quieres que le haga (32).

No hacen falta más indicios para destacar la insolidaridad de los ricos, que contrasta, precisamente, con la solidaridad entre los segadores evocada al final del cuento. Al separarse éstos de su compañero enfermo, que va para la ciudad a cuidarse en un hospital, le entregan un pequeño fajo con billetes, "un ahorro" que han hecho entre todos para sufragar los gastos del ahora ya no anónimo compañero en la miseria:

Llegaron al puente. "El Quinto" andaba apoyado en un palo medio a rastras. Zito Moraña y Amadeo le ayudaban por turno.

$-¿$ Qué tal? Ahora coges la carretera y te presentas en seguida en la ciudad.

- Si llego.

-No has de llegar. Mira, los compañeros y yo hemos hecho... un ahorro. Fs poco, pero no te vendrá mal. Tómalo.

Le dio un fajito de billetes pequeños. dos.

-Os lo acepto porque... Yo no sé... muchas gracias. Muchas gracias, Zito y to-

"El Quinto" estaba a punto de llorar, pero no sabía o lo había olvidado.

-No digas nada, hombre.

Les dio la mano largamente a cada uno.

-Adiós, Zito; adiós, Amadeo; adiós, San Juan; adiós, Conejo.

-Adiós, Pablo, adiós.

Hacía quince días que habían aprendido el nombre del "Quinto" (32).

7.- Véase J. L. Martín Nogales, op. cit., p. 137. 
No sin motivo, estos últimos rasgos, la solidaridad creciente por un lado y la cada vez mayor cercanía e intimidad entre los segadores por otro, se nos presentan predominantemente, primero, por boca de los propios implicados y, segundo, en forma de diálogos entre dos y varios interlocutores. La misma técnica de presentación de los hechos manifiesta lo que significan estas intervenciones: los implicados se comunican. Si el narrador hubiese presentado las mismas circunstancias distanciadamente y con sus palabras, el efecto expresivo hubiese sido notablemente menos intenso y entrañable.

\section{Figuras planas, estáticas y colectivas}

Desde el punto de vista de la concepción de las figuras, ni El Quinto ni las demás figuras son "redondas" en términos de E. M. Forster, es decir, no son figuras polifacéticas de las que el lector vaya conociendo muchos rasgos individualizadores y de las que puedan predecirse las reacciones. Son más bien figuras planas y fragmentarias, de las que se destacan vagamente dos o tres rasgos tipificadores como la procedencia, la pobreza, la ingenuidad y, ante todo, la solidaridad en contraste con el egoísmo de los ricos. En el caso de El Quinto se añade la poca locuacidad, la incomunicación, el mutismo, la gratitud. De ninguno se nos suministran datos acerca de su aspecto externo, si exceptuamos la "barba" en la mano de Amadeo, la forma de liar el cigarillo de San Juan y algún que otro detalle más. Pero es suficiente para la historia y la problemática que pretende evocar el autor, puesto que ésta es predominantemente interna en el sentido de que el cambio de la situación insostenible depende en gran parte de las actitudes y decisiones que asuman las figuras.

Más planas aún son las figuras del otro grupo. No sabemos detalles externos de ninguno; Aldecoa se limita a insinuar su egoísmo y su falta de solidaridad mediante algunas réplicas significativas. El único dato es el nombre de uno de ellos, Martín, y la función del otro, el alcalde. Es difícil averiguar si esta parquedad caracterizadora es resultado de la antipatía que siente el autor hacia este grupo o si es fruto exclusivamente de la economía del género.

Aparte de que la parquedad descriptiva es una característica de todas las figuras de cuentos en general, máxime si son de la escasa extensión de éste, en el caso de las figuras de Seguir de pobres se convierte en un recurso altamente expresivo. Hace más palpable el abandono, la miscria y la "condena" que significa la vida para estos hombres sentenciados a "seguir de pobres". Se suministra un mínimo imprescindible de información caracterizadora, sugiriéndose, así que permanecen en un casi-anonimato, o, con otras palabaras, no llegan a ser personas individualizadas con las capacidades precisas para urdir ni la más inofensiva protesta. Esta última circunstancia se subraya, además, por el hecho de que todas las figuras son estáticas en el sentido de que no cambian de parecer y forma de actuar durante todo el cuento. No se rebelan contra su destino, no buscan remediar su miseria de ninguna forma $y$, entre líneas, el autor nos hace ver que con estas nobles actitudes no habrá otra salida que "seguir de pobres". También es digno de reseñar el hecho de que no haya una figura principal propiamente dicha; ni Zito, como líder del grupo, ni El Quinto, que es un tipo de "antihéroe", adquieren papel de protagonistas destacados. No le interesaba al autor destacar a ninguno en particular, sino presentarlos en conjunto como figura colectiva y, por tanto, representantes de una problemática común. La intención se revela desde el mismo título del cuento, en el que se anuncia ya la suerte de los que deben "seguir de pobres".

\section{Las configuraciones}

Aunque se deba volver en este apartado a situaciones y episodios ya comentados con anterioridad, compensa insistir en ellas, ahora bajo el prisma de las figuras que se observan 


\section{KURT SPANG}

formando un conjunto pasajero en las diversas secuencias del cuento, dado que la misma configuración posec un valor expresivo propio.

Se inicia el cuento con los cinco hombres juntos ya: "En la cuadrilla van cinco hombres. Cinco hombres solos" (26). En una especie de prospección ya se adelanta, aunque todavía no en su plenitud, la situación final de la unión solidaria de los cinco hombres, pero sutilmente se insiste también en un doble aislamiento. En la breve retrospección posterior que ya cité con anterioridad, se revela la forma en la que los cuatro hombres se convirtieron en un grupo de cinco; aquí queda todavía más destacada la separación y el aislamiento de un quinteto que es más bien un cuarteto más uno. Primer indicio, tratan al Quinto de usted y, segundo indicio, no poseen objetivos comunes:

- ¿Usted qué hará cuando acabe esto?

"FI Quinto" encoge una pierna y duda.

$-i$ Yo?

-Nosotros volveremos para la tierra.

-Ya veré.

Y entre cllos, entre los cuatro y "El Quinto", el corazón de la comunidad naufraga (28).

La siguiente secuencia aporta una innovación significativa en cuanto a la configuración. Ya no tenemos que ver con este grupo único de los segadores y su problemática, ahora el panorama cambia radicalmente. Se enfrentan -como vimos- dos grupos sociales diametralmente opuestos desde la perspectiva de la problemática evocada. Aldecoa no plantea el enfrentamiento abiertamente en términos de oprimidos-opresores, pero no deja ninguna duda acerca de la provocativa e indignante discrepancia entre los dos, ni oculta por cuál de los dos siente simpatías8.

La nueva configuración refleja el dualismo entre la gente del pucblo en general y los ri$\cos$ labradores en particular, por un lado, y los segadores, por otro. Aldecoa nos introduce con precaución c intenciones implícitas: el grupo de segadores se acerca al pueblo y se encuentra con los niños:

En la mitad [de la plaza] está el pilón; en él juegan los niños. Al verlos a los cinco parados y ensimismados, los niños se les acercan a una distancia de respeto y prudencia. Los segadores, como los gitanos, pueden robar criaturitas para venderlas en otros pueblos (28-29).

¿Qué discrepancia entre la inocencia e inocuidad de los segadores y la fama que les precede en el pueblo! La situación que se describe a continuación es la de un humilde y servil acercamiento, por un lado, y cl soberbio y calculador recibimiento, por otro:

-¿Qué, Martín, hay pajar para cinco hombres?

Hay, pero no paja.

-Da igual. ¿A cuántos nos necesita usted?

-(...) El jornal, el de siempre.

-Ya aumentará usted una pesetilla.

Están los tiempos malos, pero se ha de ver (29).

EI narrador aprovecha esta circunstancia para dejar clara su propia actitud y salir en defensa de los segadores:

8.- Ibíd., p. 142. 
Precisamente están los tiempos malos. No se marcha la gente de su tierra porque estén buenos, ni porque la vida sea una delicia, ni porque los hijos tengan todo el pan que quieran (29).

El grupo de los del pueblo aumenta en dos ocasiones: al principio, apareciendo la única figura femenina participante del cuento, la mujer de Martín, que mira a los segadores como si fuesen bichos de feria:

En la cocina les dan un trozo de tocino a cada uno, pan y vino. La mujer de Martín les contempla desde una silla (29).

La segunda y brevísima secuencia presenta dos configuraciones que condensan la problemática del cuento en un espacio mínimo; es una especie de rótulo y resumen de la situación de las figuras y de la temática de la historia. Por un lado, en seis renglones se presenta la ingenuidad y el contento de los segadores al haber encontrado trabajo, y en otras siete líneas se les contrapone el desprecio de los agricultores ricos que se aprovechan de ellos (ef. 30).

La tercera secuencia gira casi exclusivamente alrededor de los segadores; sólo en una breve modificación se añade la figura del médico, que también implica una fuerte oposición en la que se contrasta no tanto la diferencia de riqueza como la de formación y saber. Como ya vimos con más detalle, se confronta la ingenua superstición y los remedios rupestres de los segadores con la altanera actitud intelectual del médico que ridiculiza las afirmaciones de Zito: "-Cuanto más ignorantes, más queréis saber. ¿Qué me vas a decir tú?" (31). Aunque tenga razón y sabiendo que son supersticiosos, es la forma de tratamiento que aquí es reveladora y marca la actitud de distancia y desprecio entre médico y segadores.

$\Lambda$ pesar de que en esta misma secuencia en el pueblo la configuración siga siendo de cinco, en determinado momento se convierte nuevamente en "cuatro más uno" cuando El Quinto enferma y se queda solo en el pajar. Ahora bien, es llamativa la diferencia entre este aislamiento y el del principio del cuento, puesto que vemos a los demás preocupados por él y cuidándole. Zito le deja su manta y le pregunta:

-¿Qué tal ahora?

-Bien, no te preocupes.

$-i$ No me he de preocupar? Has venido con nosotros y no te vas a poder marchar

La solidaridad ha nacido y se está consolidando, porque, cuando en la última secuencia el quinteto se convierte otra vez en cuarteto, ya no existe el "náufrago" del que nos habló el narrador al principio: "Mira, los compañeros y yo hemos hecho... un ahorro. Es poco, pero no te vendrá mal" (32). La despedida es una separación de un miembro de un grupo formado por cinco, ya está integrado El Quinto y parece que no es lo mismo cuatro más uno que cinco menos uno.

\section{El reparto y las figuras aludidas}

Una palabra, finalmente, acerca del reparto, es decir, de la totalidad de las figuras del cuento y de las figuras aludidas. El número de figuras participantes es relativamente reducido; además de los cinco hombres del grupo de los segadores hay que añadir los labradores del pucblo, de los que sólo dos participan efectivamente: Martín y el alcalde; luego está la mujer de Martín, es decir, un total de nueve figuras adultas y un número no fijado de niños, tanto presentes y participantes como aludidos, en el caso de los hijos de los segadores. Es significativa la circunstancia de que ambos grupos funcionen como colectivos; tanto los segadores como los agricultores son integrantes representativos de dos estamentos de la gente 


\section{KURT SPANG}

del campo, y como tales los trata $\mathrm{el}$ autor formulando la crítica del inmovilismo de la socicdad rural. Muy probablemente, el número reducido de figuras refuerce la impresión de marginación y soledad que pretende añadir el autor, aunque los dos grupos tampocọ pierden así su representatividad y la posibilidad de una generalización de la problemática plasmada.

Muy expresiva resulta la evocación de las figuras aludidas en este cuento. Baste recordar el pasaje en el que el narrador evoca los recuerdos nostálgicos de los segadores acordándose de la mujer y los hijos allá en los pueblos, donde tampoco hay más remedio que "seguir de pobres"; sin embargo, ello no es obstáculo para que estos hombres los recuerden con cariño. Lo evoca Aldecoa magistralmente a través del estilo directo libre, con los recursos de repetición, tan frecuentes, incluso predominantes, en este cuento y con un comentario aparentemente lingüístico, pero en realidad lleno de crítica amarga de la situación. Fstos detalles también revisten importancia en la caracterización de El Quinto, porque ponen de relieve el hecho de que éste no tenía a nadic a quien recordar hasta que no se produjera la incorporación solidaria en el grupo de los segadores. Los segadores hablan así:

-La mujer habrá terminado de trabajar en el pañuelo de ticrra que hemos arrendado tras de la casa. Los chavales estarán dándole vueltas al pucherillo.

Una larga pausa y la vuelta.

-Los chavales le estarán sacando brillo al puchero. La mujer saldrá a trabajar el pañuclo de tierra que hemos arrendado tras de la casa.

Dicen la mujer, los chavales, el que se fue de las calenturas, el que vino por San Juan de hará tres años. No poseen con la brutal terquedad de los afortunados y hasta parece que han olvidado en los rincones de la memoria los posesivos débiles de la vida. Están libres (27). 